\title{
2. INTERNET OF THINGS (IOT)
}

Das Internet der Dinge bzw. Internet of Things (kurz IoT) erlebt seit Jahren einen regelrechten Boom, der sich sowohl auf technologischer als auch auf betriebswirtschaftlicher Ebene abspielt. Diese Entwicklung ist Teil des aktuell stattfindenden technologischen Wandels in einer immer stärker vernetzten und digitalen Welt. So verbirgt sich unter dem Sammelbegriff IoT eine Vielzahl an Technologien und konkreten Anwendungen. Bekannte Beispiele finden sich in den Kategorien Smart Home bzw. Smart City, im Automotive-Bereich oder im sogenannten Industrial IoT.

Das Internet der Dinge bezeichnet allgemein die Verknüpfung eines physischen Objekts bzw. Gegenstands (thing) mit einer digitalen Repräsentation in einer vernetzten Struktur, die dem Internet ähnlich ist. Man kann sich also vorstellen, dass ein solch generischer Begriff viel Raum zur Interpretation lässt und nur schwer abgegrenzt werden kann. Die Übergänge sind meist fließend und die Art der Betrachtung auf dieses hoch spannende Themenfeld ist vielseitig.

So ergeben sich aus technischer Sicht Fragestellungen bzgl. Datenmanagement und Datensicherheit, da im Internet der Dinge große Mengen sensibler Daten aus der physischen Welt erhoben und ausgetauscht werden. Betriebswirtschaftliche Überlegungen beleuchten hingegen die Chancen und Risiken von IoT-Lösungen und zeigen Strategien für Unternehmen, um mittels IoT fit für die Zukunft zu werden. Das Internet of Things wird aber auch kritisch betrachtet. So wird der aktuelle Hype hinterfragt und mögliche Auswirkungen auf Unternehmen und Gesellschaft werden analysiert.

Im Folgenden werden neben Herausforderungen der digitalen Vernetzung und zahlreichen praktischen Anwendungen auch allgemeine sowie sicherheitsrelevante IoT-Themen vorgestellt.

\section{INHALT}

\section{$2.1 \quad$ IOT IN DER DIGITALEN TRANSFORMATION}

\subsubsection{Generell}

Dr. Peter Klein | Erfolgsmodell statt Elektroschrott: 5 Designprinzipien für das Internet of Things

Werner Rieche | IoT-Plattformen: das Must-have für innovative Unternehmen

Klaus Löckel | Technologie-Trends: IoT-Projekte erfolgreich umsetzen

Tillmann Braun | Missverständnis bei neuer IoT-Datenflat der Telekom -

SIM-Karten für 10 Euro helfen Unternehmen nicht weiter

Ian Hood | 5G und NFV sind Treiber der Digitalen Transformationen

2.1.2 Der Einsatz im Unternehmen

Dirk Schlimm | Internet of Things im Automotive-Bereich

Johann Götz | IoT - Praxisbetrachtung eines europäischen Großflughafens

Vincenzo Reina | Internet of Things in der Assekuranz: Wie die Generali in Deutschland

mit ihrer Smart-Insurance-Offensive Versicherungen neu erfindet

Hermann Stolle | Agile Einführung von Standard-IoT-Lösungen bei Mittelständlern

Andrei Craciun | Referenzmodell für kleine und mittlere Unternehmen -

Internet of Things: die Technologie beherrschen

Dan Matthews | Einmal nach rechts wischen für mehr Umsatz: Wie Consumer-Technologien

der Unternehmenssoftware ein neues Gesicht verleihen

\subsection{SICHERHEIT}

2.2.1 Auf dem Gerät

Philipp Benkler | Smarte Geräte auf dem Prüfstand: Software Testing im Internet of Things (1)

Kevin Bocek | Maschinelle Identitäten \& IoT - Intelligente Geräte ohne Schutz

von maschinellen Identitäten sind ein großes und wenig verstandenes Risiko

Erik Donner | Industrie 4.0 - Sicherheitstipps für das IoT der Fertigung

2.2.2 Mit Cloud-Anbindung

Johannes Wagmüller | Mit einer Box rein in die Cloud - und zurück

James LaPalme | Mit Cloud-Verschlüsselung Vertrauen schaffen

Dr. Hubert Jäger | Wie die Sealed Cloud sichere IoT-Angebote ermöglicht

Michael Morton | IT-Integration - Erfolgsfaktor der Digitalisierung 